OPEN ACCESS

Edited by:

Simone Ferrari

Sapienza Università di Roma, Italy

Reviewed by:

Zonghua Wang,

Fujian Agriculture and Forestry

University, China

Yusuke Saijo

Nara Institute of Science and

Technology, Japan

*Correspondence:

Hongli LuO

hlluo@hainu.edu.cn

Chaozu He

czhe@hainu.edu.cn

${ }^{\dagger}$ These authors have contributed equally to this work.

Specialty section

This article was submitted to

Plant Microbe Interactions,

a section of the journal

Frontiers in Microbiology

Received: 06 June 2017 Accepted: 15 December 2017

Published: 04 January 2018

Citation:

Wang Q, An B, Hou X, Guo Y, Luo H and He C (2018) Dicer-like Proteins Regulate the Growth, Conidiation, and

Pathogenicity of Colletotrichum

gloeosporioides from Hevea

brasiliensis. Front. Microbiol. 8:2621.

doi: 10.3389/fmicb.2017.02621

\section{Dicer-like Proteins Regulate the Growth, Conidiation, and Pathogenicity of Colletotrichum gloeosporioides from Hevea brasiliensis}

\author{
Qiannan Wang ${ }^{\dagger}$, Bang $\mathrm{An}^{\dagger}$, Xingrong Hou, Yunfeng Guo, Hongli Luo* and Chaozu He* \\ Hainan Key Laboratory for Sustainable Utilization of Tropical Bioresources and College of Biology, Institute of Tropical \\ Agriculture and Forestry, Hainan University, Haikou, China
}

Colletotrichum gloeosporioides from Hevea brasiliensis is the hemibiotrophic fungi which could cause anthracnose in rubber trees. Dicer like proteins (DCL) were the core enzymes for generation of small RNAs. In the present study, the knocking-out mutants of two dicer like proteins encoding genes of $C$. gloeosporioides were constructed; and functions of two proteins were investigated. The results showed that DCL play important roles in regulating the growth, conidiation and pathogenicity of $C$. gloeosporioides; and there is a functional redundancy between DCL1 and DCL2. Microscopy analysis and DAB staining revealed that loss of penetration ability into the host cells, instead of the decreased growth rate, was the main cause for the impaired pathogenicity of the $\Delta \mathrm{Dcl} 1 \Delta \mathrm{Dcl} 2$ double mutant. Proteomics analysis suggested that DCL proteins affected the expression of functional proteins to regulating multiple biological processes of $C$. gloeosporioides. These data lead to a better understanding of the functions of DCL proteins in regulating the development and pathogenesis of C. gloeosporioides.

Keywords: Colletotrichum gloeosporioides, dicer like proteins, conidiation, pathogenicity, proteomics analysis

\section{INTRODUCTION}

Rubber tree (Hevea brasiliensis) is one of important tropic economic crops which serve the primary resource of nature rubber. The anthracnose of rubber tree caused by Colletotrichum led to serious economic losses in Hainan province, the main rubber planting area of China. In the preliminary work, we found that Colletotrichum gloeosporioides is the main pathogen of anthracnose. To explore the molecular mechanism of its pathogenicity, the genome of C. gloeosporioides from was sequenced (data unpublished). Colletotrichum species are common plant pathogens which have a hemibiotrophic lifestyle, meaning that they are biotrophic at initial stage of infection to their host cells and necrotrophic at later phase (Koeck et al., 2011; O'Connell et al., 2012). It is vital for pathogens to overcome the plant immune system at the biotrophic stage. Effector proteins were thought to play as molecular weapons to suppress the plant immunity (Jones and Dangl, 2006; Göhre and Robatzek, 2008; Rafiqi et al., 2012). When interact with the host plant, Colletotrichum higginsianum could secret a lot of effector proteins by appressoria and intracellular hyphae to manipulate the plant physical processes to ensure its successful evasion (Stephenson et al., 2000; Kleemann et al., 2012; Pumplin and Voinnet, 2013). 
Small RNAs, about 20-30 nucleotide long, are small noncoding RNAs found in living cells. Small RNAs could regulate the gene expression at both posttranscriptional and transcriptional levels, which is known as gene silencing (PTGS) (Matzke and Matzke, 1995), quelling (Romano and Macino, 1992), and RNA interference (RNAi) (Bass, 2000). There are three major classes of small RNAs identified in eukaryotes by now: small interfering RNA (siRNA), microRNA (miRNA) and piwi-interacting RNA (piRNA) (Carthew and Sontheimer, 2009; Moazed, 2009), and each class of small RNAs has diverse functions. In animals and plants, small RNAs are proved to play vital roles in regulating multiple biological processes, including morphogenesis, hormone signaling and stress responses (Bartel, 2004; Rubio-Somoza et al., 2009; Mendell and Olson, 2012). In fungi, small RNAs also play multiple biological functions, including regulation of heterochromatin formation in Schizosaccharomyces pombe (Volpe et al., 2002), controlling of transposon in Neurospora crassa (Nolan et al., 2005), and mediating defense against virus in Aspergillus nidulans (Ding and Lu, 2011). Recent works show that the fungal pathogens could also manipulate the immunity system of the host plant by generating small RNAs. Weiberg et al. (2013) found that Botrytis cinerea (a necrotrophic pathogen) could secret small RNAs into the host plant, hijack the host Argonaute (AGO) proteins to decrease the plant immunity. Dicer like proteins (DCL) are important for generation of small RNAs in living cells (MacRae et al., 2006; Xue et al., 2012). Many animals and fungi have two Dicer-like genes $(\mathrm{Dcl})$, and plant have four or six Dicer-like genes. In the human endothelial cells, microRNAs regulate the gene expression in a dicer dependent manner (Suárez et al., 2007). In Drosophila cells, DCL1 and DCL2 play distinct roles in processing miRNA precursors and siRNA precursors (Lee et al., 2004). In Arabidopsis thaliana, DCLs play multiple roles in regulating biological processes, such as flowering process (Schmitz et al., 2007) and virus induced host silencing (Blevins et al., 2006). In fungi, DCLs of B. cinerea are proved to be involved in vegetative growth and processing of small RNAs (Weiberg et al., 2013). The functions of DCLs in C. gloeosporioides are still unclear. Therefore, in the present study, two $\mathrm{Dcl}$ genes in C. gloeosporioides were knocked out and their functions in regulating growth, conidiation and pathogenicity were investigated.

\section{MATERIALS AND METHODS}

\section{Fungal Strains and Culture Conditions}

Colletotrichum gloeosporioides was isolated from the $H$. brasiliensis with Anthracnose; furthermore, the genome of the C. gloeosporioides was sequenced for exploration of possible mechanism of pathogenicity (data unpublished). The C. gloeosporioides was used as recipient strain for the transformation experiments and as a WT control. All the C. gloeosporioides strains were grown on potato dextrose agar (PDA) at $28^{\circ} \mathrm{C}$.

\section{Vector Constructions}

Vector pCB1532 carrying the acetolactate synthase gene (SUR) cassette from M. oryzae (Yang et al., 2013) conferred resistance to chlorimuron ethyl (a sulfonylurea herbicide) and pKOV21 carrying the hygromycin phosphotransferase gene (HPH) conferred resistance to hygromycin were used to construct the replacement vectors. The replacement vector for Dcl1 was designed as described in Figure 1A: the $5^{\prime}$ flanking region and $3^{\prime}$ region of the $D c l 1$ nucleotide were amplified by use of the primer pairs $1 / 2$ and $3 / 4$, respectively; then the two fragments were excised with EcoRI/HindIII and XbaI/EcoRI respectively and ligated into vector pCB1532 to construct the replacement vector. The replacement vector for $\mathrm{Dcl} 2$ was designed as described in Figure 1B: the $5^{\prime}$ flanking region and $3^{\prime}$ region of the $D c l 2$ nucleotide were amplified by use of the primer pairs 5/6 and $7 / 8$, respectively; then the two fragments were excised with EcoRI/HindIII and XbaI/EcoRI respectively and ligated into vector pKOV21 to construct the replacement vector. The two deletion vectors were excised with EcoRI before transformation of the C. gloeosporioides strain. Vector pBS-NEO carrying the Neomycin phosphotransferase gene (NPTII) conferred resistance to Geneticin (G418) was used to construct the complementation vector. To get the complementation vector, a $6.4 \mathrm{~kb}$ fragment containing Dcl1 the sequence together with a $1.4 \mathrm{~kb}$ upstream nucleotide and a $5.9 \mathrm{~kb}$ fragment containing Dcl2 the sequence together with a $1.3 \mathrm{~kb}$ upstream nucleotide were amplified by using the primers 21/22 and 23/24; then the two fragments were excised with $\mathrm{XbaI} / \mathrm{BamHI}$ and SacII/XbaI respectively and ligated into the vector pBS-NEO (Figure S2A). The complementation vector was excised with $\mathrm{XbaI}$ before transformation of the double deletion mutant strain.

\section{Transformation of C. gloeosporioides, PCR Diagnosis, and Single Conidia Purification}

Conidia was inoculated into the $200 \mathrm{~mL}$ potato broth to make the initial number of conidia to $10^{5}$ conidia $\mathrm{ml}^{-1}$ and then cultured at $28^{\circ} \mathrm{C}, 150 \mathrm{rpm}$ for $24 \mathrm{~h}$. Then the mycelium were collected with the nylon membrane, washed two times with $1 \mathrm{M}$ sorbitol and transfer to the $1 \mathrm{M}$ sorbitol containing the $10 \mathrm{mg} \mathrm{ml}^{-1}$ lysing enzyme (Sigma-Aldrich). Then the mycelium was incubated at $28^{\circ} \mathrm{C}, 100 \mathrm{rpm}$ for $3 \mathrm{~h}$ to catalyze the cell wall. After that, the protoplast were filtered with nylon membrane and collected by centrifugation with $2,000 \mathrm{rpm}$ at $4^{\circ} \mathrm{C}$, washed two times and resuspened with STC buffer (1 M sorbitol, $50 \mathrm{mMTris}-\mathrm{Cl}, 10 \mathrm{mM} \mathrm{CaCl}_{2}, \mathrm{pH} \mathrm{7.4)}$ to the final concentration of $10^{8} \mathrm{ml}^{-1} \mathrm{CFU}$. For the transformation, 100 $\mu \mathrm{L}$ linearized replacement vector was added into the $200 \mu \mathrm{L}$ protoplast and the mixture was incubated on ice for $20 \mathrm{~min}$. Then $1 \mathrm{ml} 40 \%$ PEG dissolved in STC buffer was added into the protoplast mixture and placed for $20 \mathrm{~min}$ at $28^{\circ} \mathrm{C}$. After the transformation, $5 \mathrm{~mL}$ liquid regeneration medium $\left(1 \mathrm{~g} \mathrm{~L}^{-1}\right.$ yeast extract, $1 \mathrm{~g} \mathrm{~L}^{-1}$ casein, $6 \mathrm{M}$ sucrose) was added into the protoplast and cultured at $28^{\circ} \mathrm{C}, 100 \mathrm{rpm}$ for $4 \mathrm{~h}$. Then the regenerated protoplast was transfer into the regeneration medium with $1 \%$ agar at about $50^{\circ} \mathrm{C}$, mixed gently and spread on the petri dish. After the agar concreted, same volume of regeneration medium with $1 \%$ agar containing $100 \mu \mathrm{g} \mathrm{ml}^{-1}$ chlorimuron ethyl or $200 \mu \mathrm{g} \mathrm{ml}^{-1}$ hygromycin was spread on the upper level to select the transformants. For generation of the double mutants, the Dcl1 deletion mutants were used as the recipient strain to conduct the protoplast preparation and 
transformation to delete $D c l 2$. The chlorimuron ethyl-resistant or the hygromycin-resistant strains were isolated and analyzed by PCR with the primer pairs as showed in Figure 1, which are diagnostic for homologous integration of $5^{\prime}$ part and $3^{\prime}$ part. Then the correct transformants were purified by single conidia isolations. Single conidial isolates were obtained by spreading $100 \mu \mathrm{L}$ of conidial suspension $\left(10^{4}\right.$ conidia $\left.\mathrm{mL}^{-1}\right)$ on Malt extract agar medium (BD, USA) plates containing $100 \mu \mathrm{g} \mathrm{mL}^{-1}$ chlorimuron ethyl or $300 \mu \mathrm{g} \mathrm{mL}^{-1}$ hygromycin. For generation of the complementation mutants, the Dcl1 and Dcl2 double deletion mutants were used as the recipient strain to conduct the transformation. Regeneration medium containing $200 \mu \mathrm{g}$ $\mathrm{ml}^{-1} \mathrm{G} 418$ was used to select the transformants. PCR with the primer pairs 21/22 and 23/24 were used for integration diagnosis. After that, Single conidial isolation was conducted to purify the transformants.

\section{Southern Blot Analysis}

The genomic DNA of the WT and the mutants were extracted and excised with EcoRI. The DNA probes was amplified and labeled by digoxin using digoxigenin-dUTP (Roche) as shown in Figure 1. The DNA band with the hybridized probe was visualized using an enzyme immunoassay and enzyme catalyzed color reaction with NBT/BCIP (Roche).

\section{Growth and Conidiation Assay}

Wild-type and the mutant strains were grown for 3 day on PDA medium and a disk of hypha with diameter of $1 \mathrm{~mm}$ was removed from the growing edge; then the disk of hypha was inoculated on the complete medium (CM) and minimal medium (MM). After culture for 5 days, the diameter was recorded and the growth rate was calculated. Conidia were harvested from C. gloeosporioides strain grown on PDA medium for 12 day, inoculated into $50 \mathrm{~mL}$ liquid CM medium to the final concentration of $10^{3} \mathrm{~mL}^{-1}$, and cultured at $28^{\circ} \mathrm{C}, 150 \mathrm{rpm}$ for desired time. Then the conidia number after incubation for 3 and 4 days were numbered under microscope.

\section{Pathogenicity Assay}

For the pathogenicity assay, conidia of the WT and the mutants were collected, washed two times with $\mathrm{ddH}_{2} \mathrm{O}$ and resuspended in a solution of $5 \%$ Sabouraud Maltose Broth (Difco) to a final concentration of $2 \times 10^{5}$ conidia $\mathrm{mL}^{-1}$. Droplets (5 $\mu \mathrm{L}$ ) of the conidial suspensions were used to inoculate the detached "light green" leaves from rubber tree Varity 73-3-97. The inoculated leaves were kept in a moist chamber at $28^{\circ} \mathrm{C}$ under natural illumination for 4 days and the disease symptoms were scored. Each treatment contained three replicates of 15 leaves and the entire experiment was repeated three times.
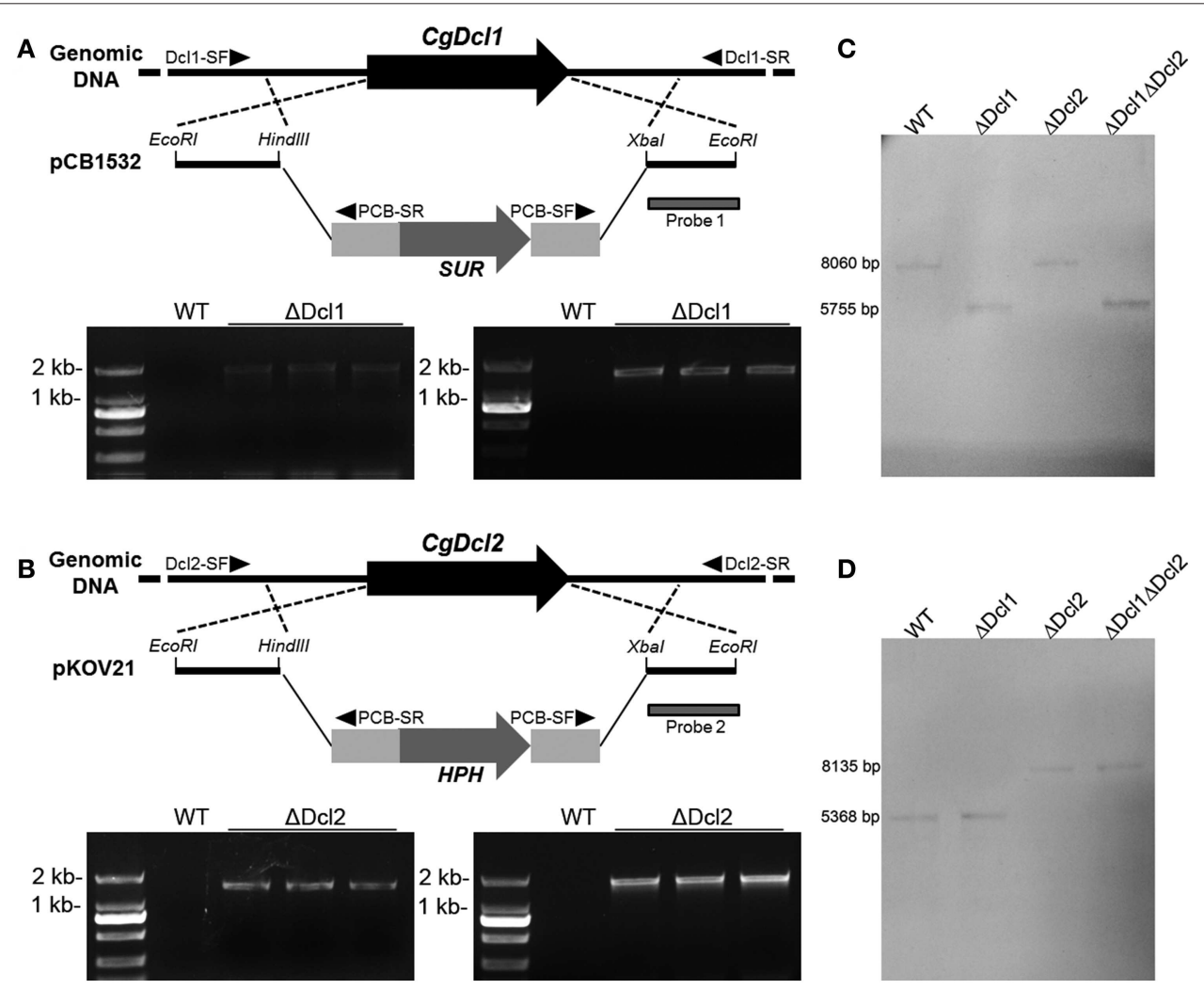

FIGURE 1 | The gene deletion strategy and PCR confirmation of Dc/1 (A) and Dcl2 (B) in C. gloeosporioides. Putative mutants were screened with diagnostic primers, which were indicated with black triangles. (C,D) Confirmation of the correct recombination of the deletion cassettes to the gene loci with Southern blot. The downstream flanking of the sequences were used as the probes for Southern blot analyses. 


\section{Penetration Ability Assay}

Conidia was prepared as mentioned above and resuspended with sterilized $\mathrm{ddH}_{2} \mathrm{O}$ to the final concentration of $2 \times 10^{5}$ conidia $\mathrm{mL}^{-1}$. Onion epidermis was harvested and put on water agar plates. Then the onion epidermis was inoculated with $20 \mu \mathrm{L}$ of conidia suspension and kept in a moist chamber at $28^{\circ} \mathrm{C}$ for desired time. After that, the infection structures were analyzed with microscope. The penetration rates of the conidia were quantified after inoculation for $12 \mathrm{~h}$. About 50 conidia were counted under the microscope, and each treatment contained three replication. Sterilized cellophane paper were put on malt extract agar medium, inoculated with $10 \mu \mathrm{L}$ of conidial suspension, and kept in a moist chamber at $28^{\circ} \mathrm{C}$ for desired time. The germination were observed by using microscope.

\section{Oxidative Burst Assay}

The rubber tree leaves were wounded with sterilized needle. Conidia of WT and mutant strains was prepared as mentioned above, resuspended with 5\% Sabouraud Maltose Broth (Difco) to a final concentration of $2 \times 10^{5}$ conidia $\mathrm{mL}^{-1}$. Droplets $(5 \mu \mathrm{L})$ of the conidial suspensions were used to inoculate the wounded leaves. The inoculated leaves were kept in a moist chamber at $28^{\circ} \mathrm{C}$ under natural illumination for $24 \mathrm{~h}$. Then the oxidative burst was analyzed by staining the leaves with DAB (SigmaAldrich) according to the protocol from Daudi et al. (2012). Wounded leaves inoculated with the only 5\% Sabouraud Maltose Broth were used as control check (CK).

\section{Proteomic Analysis}

Protein extraction, two-dimensional (2D) gel electrophoresis, image analysis and in-gel digestion were carried out according to An et al. (2016). Wild-type and the mutant strains were grown for 3 day on cellophane paper plated on the Malt extract agar medium. The hyphae were collected and disrupted in liquid nitrogen by grinding in a mortar with a pestle. Total protein were extracted with the extraction buffer containing 0.5 M Tris- $\mathrm{HCl}, \mathrm{pH} 8.3,2 \%$ (v/v) NP-40, $20 \mathrm{mM} \mathrm{MgCl}_{2}, 2 \%$ $(\mathrm{v} / \mathrm{v}) \beta$-mercaptoethanol, and $1 \mathrm{mM}$ PMSF. After removing the cell debris by centrifugation, the supernatant was extracted with an equal volume of Tris- $\mathrm{HCl}(\mathrm{pH}$ 7.8) buffered phenol. After centrifugation, proteins were precipitated from the final phenol phase with 5 vol of ice-cold saturated ammonium acetate in methanol overnight at $-20^{\circ} \mathrm{C}$. Then the proteins were collected by centrifugation and washed twice with cold saturated ammonium acetate in methanol and acetone. The precipitate was air-dried for $1 \mathrm{~h}$ at $4^{\circ} \mathrm{C}$ and then solubilized in the thiourea/urea lysis buffer containing $2 \mathrm{M}$ thiourea, $7 \mathrm{M}$ urea, $4 \%$ (w/v) CHAPS, 1\% (w/v) DTT, and 2\% (v/v) carrier ampholytes of $\mathrm{pH}$ 3-10. For two-dimensional (2D) gel electrophoresis, aliquots of $650 \mu \mathrm{g}$ of proteins resolved in $340 \mu \mathrm{L}$ of sample buffer (7 M urea, $2 \mathrm{M}$ thiourea, 4\% (w/v) CHAPS, 1\% (w/v) DTT, $2 \%(\mathrm{v} / \mathrm{v})$ carrier ampholytes ( $\mathrm{pH} 3-10)$, and $0.001 \%$ $(\mathrm{w} / \mathrm{v})$ bromphenol blue) were used to rehydrate gel strips (Immobiline DryStrip pH 4-7, $18 \mathrm{~cm}$; GE Healthcare) for $16 \mathrm{~h}$. The first-dimensional IEF, equilibration of the IPG strips and the second-dimensional separation were conducted with the Ettan IPGphor III and the Ettan DALTsix (GE Healthcare) systems according to the manufacturer's instructions. Protein spots whose expression levels changed by $>2$-fold were excised for protein identification using a MALDI-TOF/TOF mass spectrometer (Ultrafle Xtreme; Bruker Daltonics, Billerica, MA, USA). MS data were uploaded to Mascot for database searching on the Matrix Science (London, UK) public website (http://www.matrixscience. com) and searched against the NCBInr protein database with BIOTOOLS software (v.3.2; Bruker Daltonics). Search parameters were set as taxonomy: Fungi; enzyme: trypsin; max missed cleavages: 1; fixed modifications: carbamidomethyl (C); variable modifications: oxidation $(\mathrm{M})$; peptide mass tolerance: \pm 100 ppm; fragment mass tolerance: $\pm 0.5 \mathrm{Da}$. In addition, a peptide charge of $1+$ and a monoisotopic mass were selected and the instrument type was set to MALDI-TOF-TOF.

\section{Quantitative RT-PCR Analysis}

Wild-type and the mutant strains were grown for 3 day on cellophane paper plated on the Malt extract agar medium. The hyphae were collected and disrupted in liquid nitrogen by grinding in a mortar with a pestle, then the RNA was extracted using TRIzol Reagent (Invitrogen). Reverse transcription was conducted with RevertAid RT Reverse Transcription Kit (Thermo Fisher) according to the manufacturer's instructions. Quantitative RT-PCR analysis was performed with the LightCycler 96 System (Roche). The beta-tubulin-1 gene was used as an endogenous control for normalization. Relative expression levels were estimated using the $2^{-\Delta \Delta \mathrm{Ct}}$ method. The primers used for quantitative RT-PCR are listed in Table S1.

\section{RESULTS}

\section{Generation of the $\Delta \mathrm{Dcl1}, \Delta \mathrm{Dcl} 2$, $\Delta$ Dcl1 $\Delta \mathrm{Dcl} 2$, and the Complementation Mutants}

Two dicer like protein (DCL) coding gene were identified in C. gloeosporioides using the genome database. The Dcl1 consists of 4,900 bp and codes a protein composed of 1564 amino acids; the Dcl2 consists of 4618 bp and codes a protein composed of 1453 amino acids. To explore their functions in C. gloeosporioides, the nucleotides of two genes were deleted by a replacement strategy as shown in Figure 1. The Chlorimuron ethyl or Hygromycin resistant colonies were analyzed for homologous integration by PCR. To verify the integration locus by PCR, primer pairs with one primer being located outside and one inside the construct were used. As shown in Figure 1, at least three mutants showed the both diagnostic fragments of $5^{\prime}$ and $3^{\prime}$ flanking region. The fragments were sequenced to ensure flawlessness. The results showed that the replacement fragments were correctly integrated into the $\mathrm{Dcl}$ loci. The knocking-out mutants were named $\Delta \mathrm{Dcl} 1$ and $\Delta \mathrm{Dcl} 2$ respectively. The results of PCR diagnosis of the double mutant $(\Delta \mathrm{Dcl} 1 \Delta \mathrm{Dcl} 2)$ showed all the diagnostic fragments. All the transformants were purified by single conidia isolation. The detection of WT nuclei of single conidia isolations was implemented by PCR with gene primer pair 17/18 and 19/20. The Southern blot assay indicated that the $\Delta \mathrm{Dcl} 1, \Delta \mathrm{Dcl} 2$, and the $\Delta \mathrm{Dcl} 1 \Delta \mathrm{Dcl} 2$ mutants all showed single 
homologous integration; and the hybrid bands were consistent with expected DNA sequence lengths (Figures 1C,D). Since the purified single conidia isolates of the three mutants showed the identical phenotypes both in growth rate and pathogenicity, only one strain of each kind of mutant was chosen for detailed studies. Complemented mutant strains were diagnosed by PCR with the primer pairs $21 / 22$ and $23 / 24$. The correct transformants were also purified by single conidia isolation.

\section{DCLs Are Involved in Vegetative Growth and Conidiation}

The $\Delta \mathrm{Dcl} 1$ and $\Delta \mathrm{Dcl} 2$ showed similar growth rate to $\mathrm{WT}$ when cultured on complete medium or minimal medium; while the vegetative growth of $\Delta \mathrm{Dcl} 1 \Delta \mathrm{Dcl} 2$ was obviously decreased compared with WT (Figure 2A). When cultured in liquid medium, $\Delta \mathrm{Dcl} 2$ generated similar amount of conidia compared with WT, while $\Delta \mathrm{Dcl} 1$ showed an obvious increase in conidiation; for the $\Delta \mathrm{Dcl} 1 \Delta \mathrm{Dcl} 2$, the conidiation was seriously impaired (Figure 2B). After reintroducing the Dcl1 and Dcl2 nucleotides back into the double deletion mutant, the growth rate of the complemented mutant strain was restored (Figure S2B).

\section{DCLs Are Required for Pathogenicity and Penetration Process}

Detached "light green" leaves from rubber tree Varity 73-3-97 were used to determine the pathogenicity of the mutant strains. As shown in Figure 3, about $80 \%$ conidia of the WT, $\Delta \mathrm{Dcl} 1$ and $\Delta \mathrm{Dcl} 2$ strains was able to invade the leaves and cause disease, and the lesion diameter were about $6 \mathrm{~mm}$ and $9 \mathrm{~mm}$ at 2 and $3 \mathrm{dpi}$. But $\Delta \mathrm{Dcl} 1 \Delta \mathrm{Dcl} 2$ completely lost the pathogenicity on the rubber leaves. The lesions produced by the complemented mutant strain
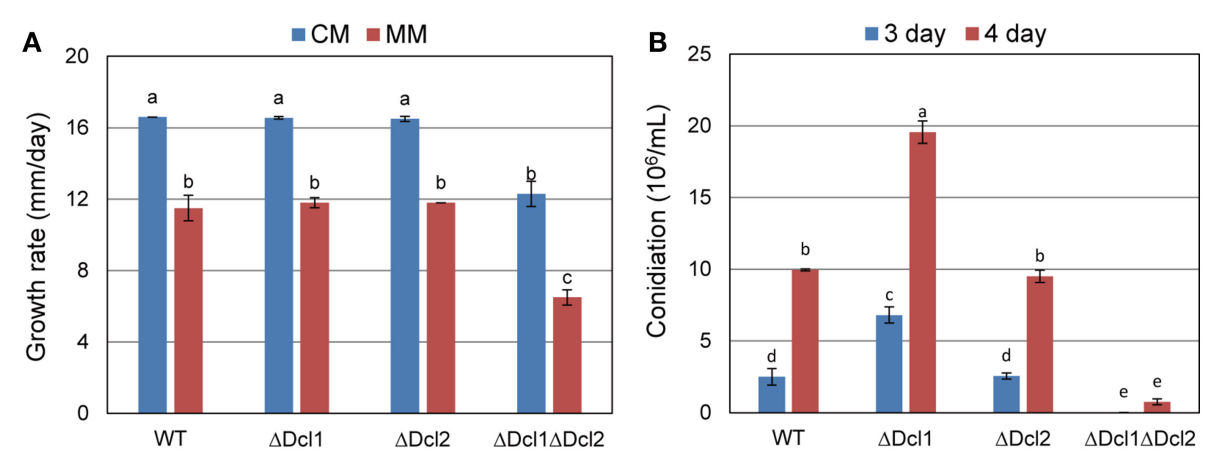

FIGURE 2 | Growth rate and conidiation of C. gloeosporioides. (A) Growth rate of WT and the mutant strains on complete medium (CM) and minimal medium (MM) for 5 days. (B) Conidation of WT and the mutant strains. Bars represent standard deviations (SD). Columns with different letters indicate significant difference $(P<0.05)$.
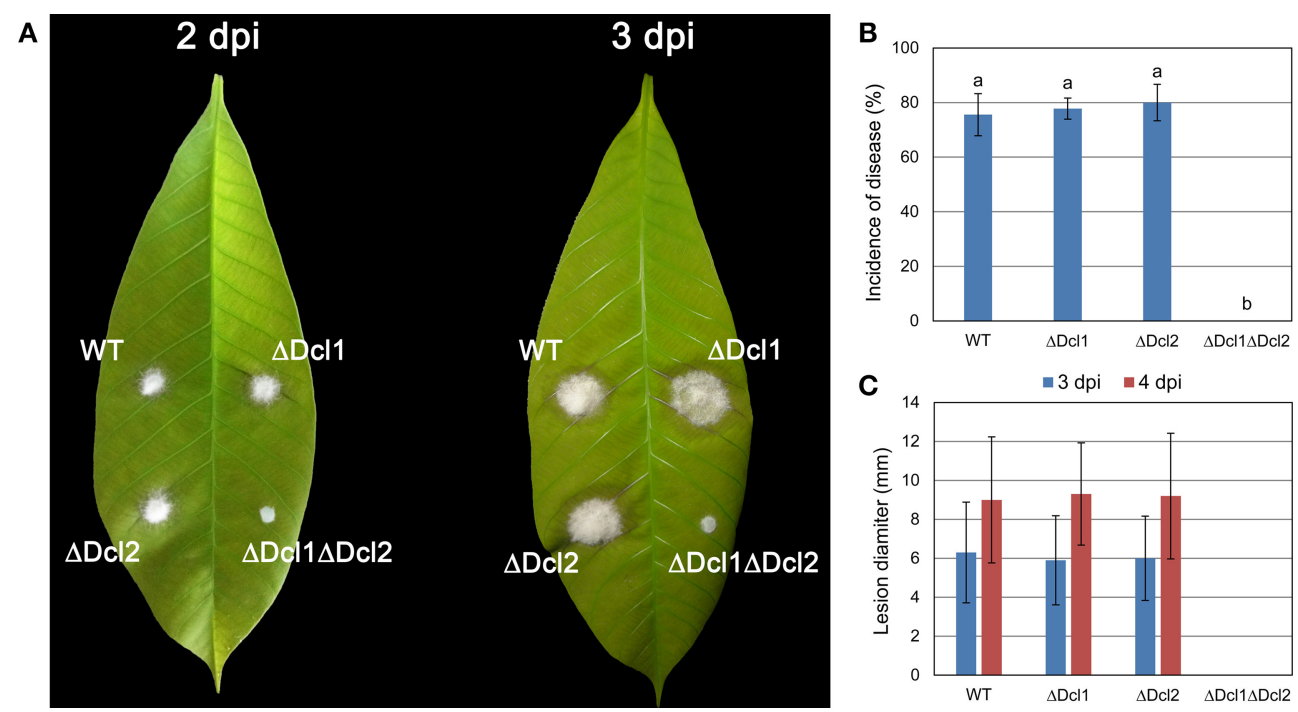

FIGURE 3 | Virulence assay on rubber tree leaves. (A) Disease symptoms of rubber tree leaves at 2 day post inoculation (dpi) and 3 dpi. (B) Mean incidence of disease of rubber tree leaves at 3 dpi. (C) Mean lesion diameters after 2 and 3 dpi. Bars represent standard deviations (SD). Columns with different letters indicate significant difference $(P<0.05)$. 

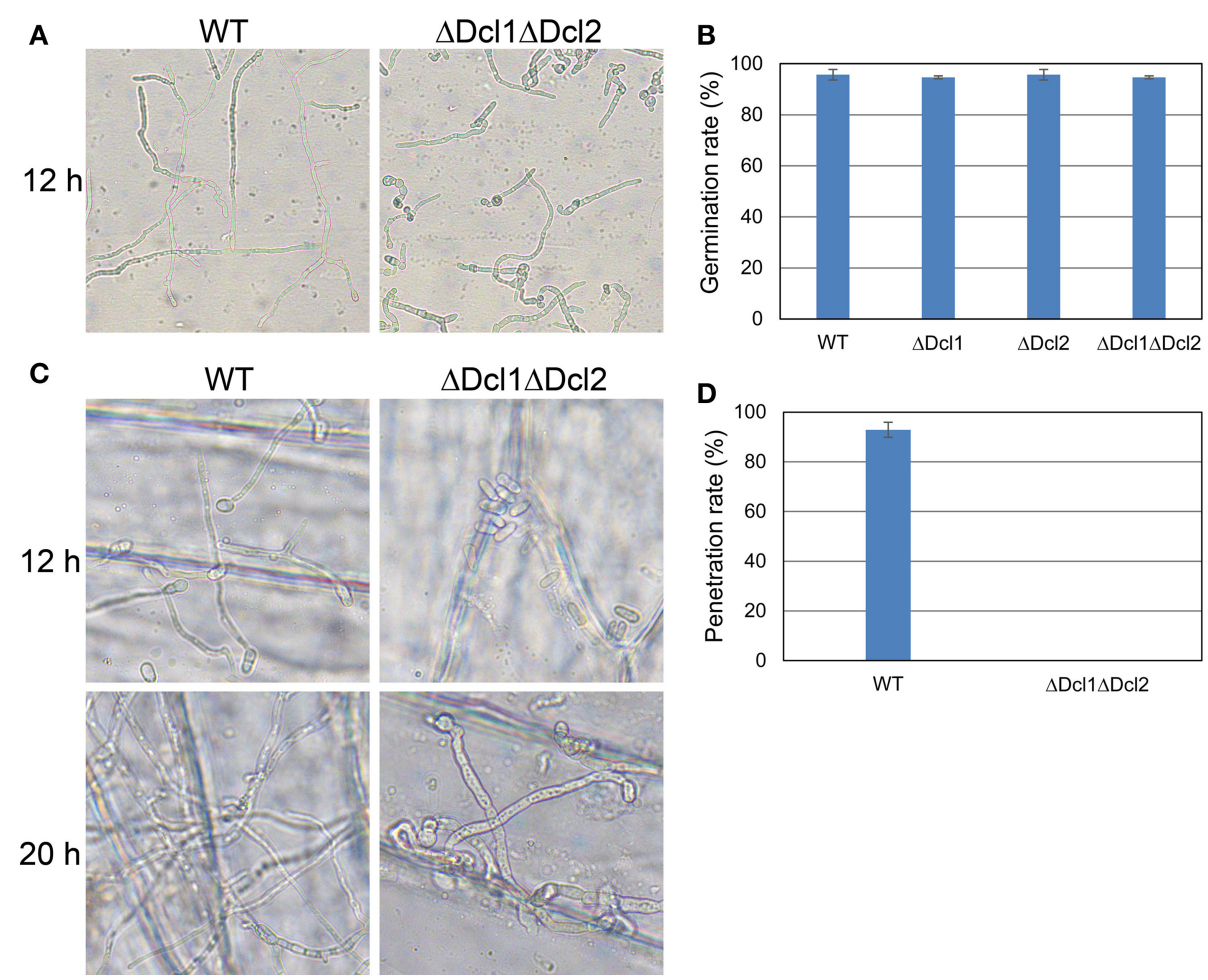

FIGURE 4 | Growth assays of WT and $\Delta \mathrm{Dcl} 1 \Delta \mathrm{Dcl} 2$ mutant on cellophane and onion epidermis. (A) Germination behavior after culture for $12 \mathrm{~h}$. (B) Germination rate of conidia of all the strains after culture for $6 \mathrm{~h}$. Bars represent standard deviations (SD). (C) Penetration assay of WT and $\Delta \mathrm{Dcl} 1 \Delta \mathrm{Dcl} 2 \mathrm{mutant}$ on onion epidermis after inoculation for 12 and $20 \mathrm{~h}$. (D) Penetration rate of conidia of WT and $\Delta \mathrm{Dcl} 1 \Delta \mathrm{Dcl} 2$ inoculated on onion epidermis for $12 \mathrm{~h}$.

were nearly similar to those produced by the WT strain (Figure S2C).

To gain a further insight, we tested the conidial germination and penetration on onion epidermis and cellophane paper. After incubation of $12 \mathrm{~h}$, all the conidia of WT germinated and penetrated into the onion epidermis; but the conidia of $\Delta \mathrm{Dcl} 1 \Delta \mathrm{Dcl} 2$ mutant did not geminated at all; after $24 \mathrm{~h}$, mycelium of WT formed complex networks in onion cells; but the conidia of $\Delta \mathrm{Dcl} 1 \Delta \mathrm{Dcl} 2$ mutant had just germinated with abnormal germ tubes (Figure 4A). When cultured on the cellophane paper, conidia of $\Delta \mathrm{Dcl} 1 \Delta \mathrm{Dcl} 2$ mutant germinated at the same rate as WT, although the growth rate was decreased (Figures 4B,C). These results suggested that the penetration ability of $\triangle \mathrm{Dcl} 1 \Delta \mathrm{Dcl} 2$ mutant was impaired. DAB staining showed that when the wounds were inoculated with the conidia of WT, the rubber leaves could generate significant oxidative burst compared with the CK (inoculated with only Maltose Broth); but that inoculated with $\Delta \mathrm{Dcl} 1 \Delta \mathrm{Dcl} 2$ mutant did not show significant increase in peroxide (Figure 5).

\section{DCLs Are Required for Generation of Functional Proteins}

A comparative proteomic analysis was conducted to identify the proteins that regulated by DCLs. The $2 \mathrm{D}$ gel electrophoresis was conducted to separate the proteins isolated from the WT and the $\Delta \mathrm{Dcl} 1 \Delta \mathrm{Dcl} 2$ mutant; approximately 1680 protein spots

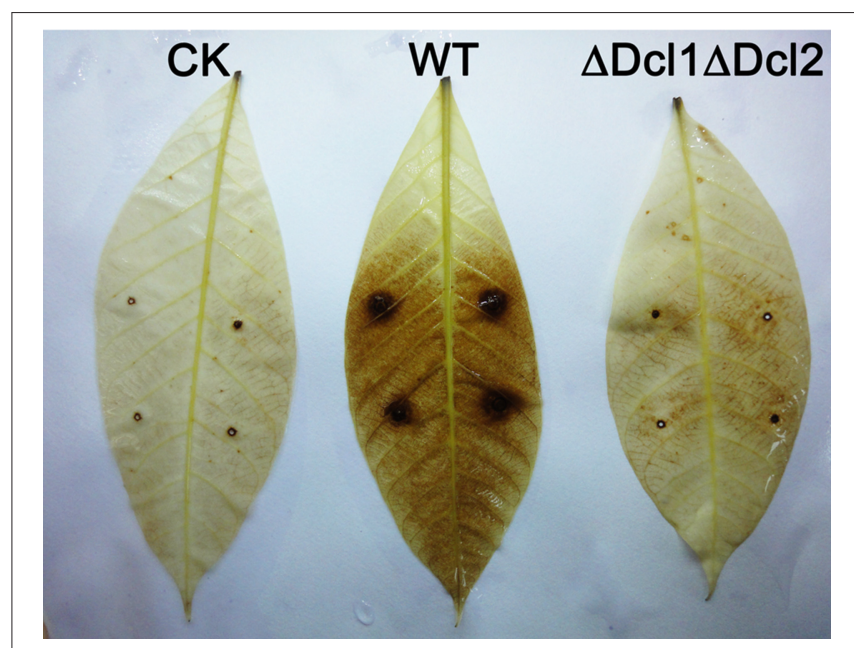

FIGURE 5 | Oxidative burst assay of rubber tree leaves inoculated with WT and $\triangle \mathrm{Dcl} 1 \triangle \mathrm{Dcl} 2$ mutant by DAB staining.

were detected on CBB-stained 2D gels. The quantitative image analysis revealed a total of 72 protein spots that showed at least 2 -fold down regulation in abundance $(\mathrm{P}<0.05)$ in the double mutant (Figure 6). Besides, only a few protein spots showed up-regulated with abundance change smaller than 2 fold in the 


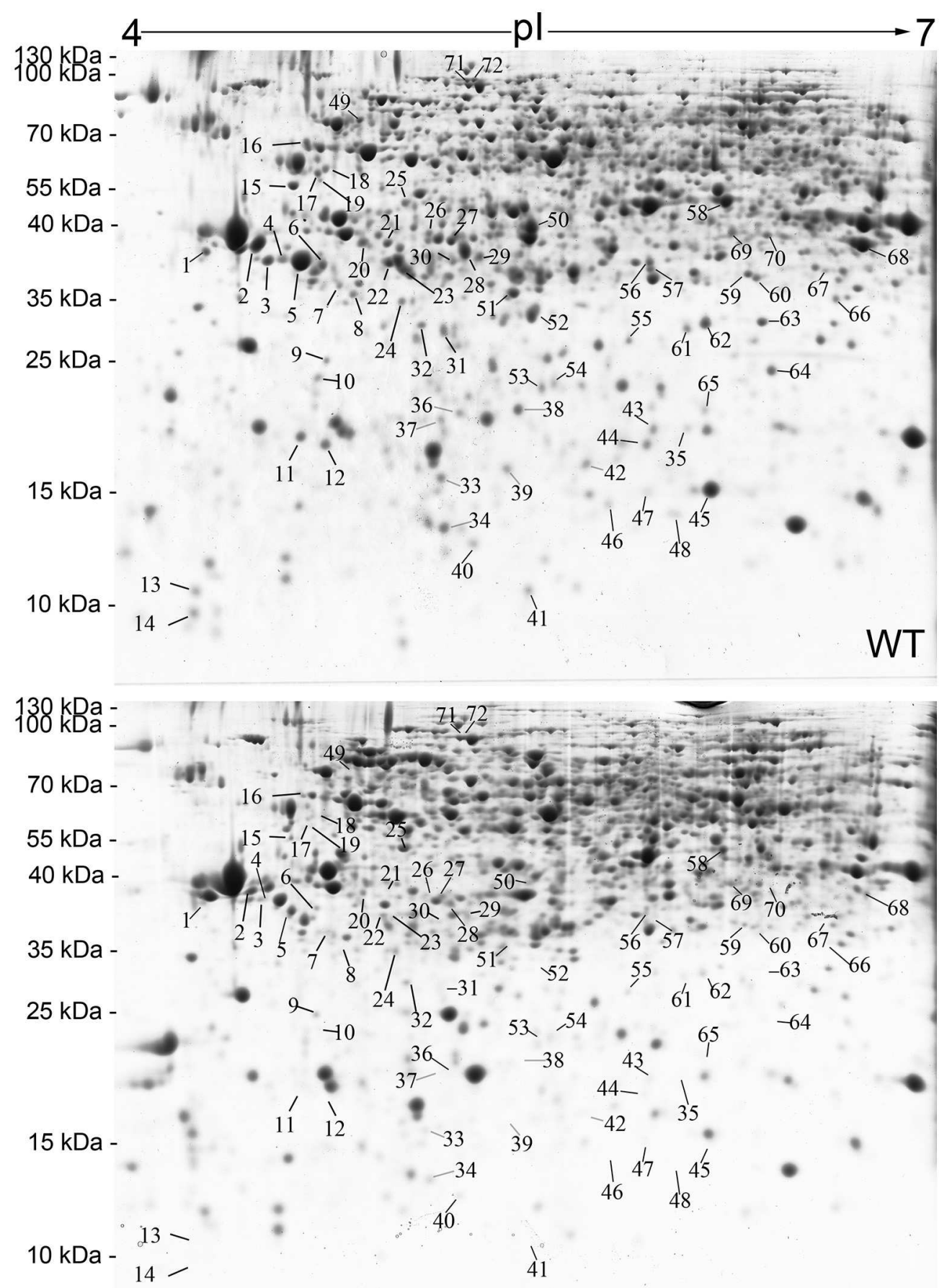

$\Delta \mathrm{Dcl} 1 \Delta \mathrm{Dcl} 2$

FIGURE 6 | Two-dimensional patterns of proteins of WT and $\Delta \mathrm{Dcl} 1 \Delta \mathrm{Dcl} 2$ mutant of $C$. gloeosporioides from $H$. brasiliensis. Arrows indicate protein spots which down regulated in abundance more than 2 -fold between WT and the mutant. The protein spots are numbered corresponding to those in Table $\mathbf{1 .}$

mutant. So only the down-regulated protein spots were further analyzed. The 72 down-regulated protein spots were excised and submitted to tandem mass spectrometry and identified by database searching with the Mascot search engine (Table 1). The identified proteins were classified into 9 functional categories based on the FunCatannotation scheme (http:// ibis.helmholtz-muenchen.de/funcatDB/), including protein synthesis, cell cycle, chemical metabolism, hydrolytic enzyme, signal transduction, transport, charpones, cell structure and unknown. 
TABLE 1 | Proteins Identified in C. gloeosporioides hyphae by quadrupole time-of-Flight tandem mass spectrometry.

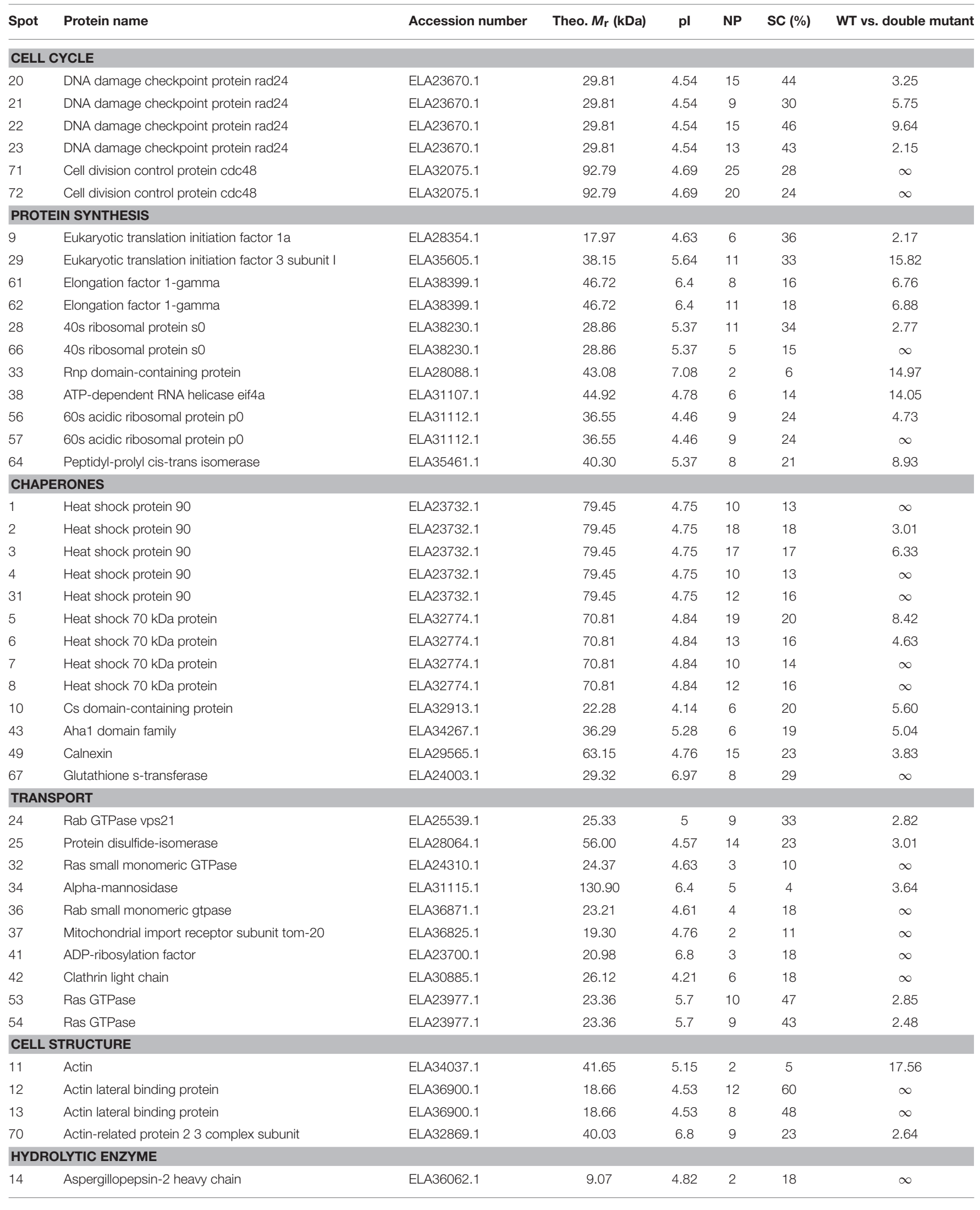


TABLE 1 | Continued

\begin{tabular}{|c|c|c|c|c|c|c|c|}
\hline Spot & Protein name & Accession number & Theo. $M_{\mathrm{r}}(\mathrm{kDa})$ & pl & NP & SC (\%) & WT vs. double mutant \\
\hline 15 & Endochitinase & ELA36416.1 & 44.79 & 4.57 & 10 & 24 & 2.46 \\
\hline 16 & Aspartic endopeptidase & ELA37088.1 & 50.57 & 4.68 & 12 & 24 & 8.65 \\
\hline 27 & Proteasome endopeptidase complex & ELA37155.1 & 39.16 & 4.11 & 14 & 33 & 1.99 \\
\hline \multicolumn{8}{|c|}{ SIGNAL TRANSDUCTION } \\
\hline 17 & Protein phosphatase & ELA27101.1 & 49.29 & 4.41 & 14 & 30 & 6.25 \\
\hline 18 & Protein phosphatase & ELA27101.1 & 49.29 & 4.41 & 9 & 17 & 6.23 \\
\hline 19 & Protein phosphatase & ELA27101.1 & 49.29 & 4.41 & 9 & 19 & 2.02 \\
\hline 26 & cAMP-dependent protein kinase regulatory subunit & ELA30315.1 & 42.08 & 4.59 & 8 & 19 & $\infty$ \\
\hline 30 & Protein phosphatase pp2a regulatory subunit a & ELA34582.1 & 69.65 & 4.56 & 11 & 12 & 7.92 \\
\hline 35 & Rho protein gdp dissociation inhibitor containing protein & ELA26991.1 & 33.50 & 6.18 & 8 & 27 & $\infty$ \\
\hline 44 & Rho protein gdp dissociation inhibitor containing protein & ELA26991.1 & 33.50 & 6.18 & 7 & 22 & $\infty$ \\
\hline 39 & Dual specificity catalytic domain containing protein & ELA30352.1 & 45.31 & 6.52 & 7 & 13 & 3.83 \\
\hline \multicolumn{8}{|c|}{ CHEMICAL METABOLISM } \\
\hline 40 & Ethyl tert-butyl ether degradation & ELA35540.1 & 12.00 & 5.01 & 7 & 70 & 2.93 \\
\hline 50 & Aldehyde dehydrogenase & ELA36993.1 & 49.96 & 6.25 & 13 & 30 & 9.06 \\
\hline 51 & Aldehyde dehydrogenase & ELA36993.1 & 49.96 & 6.25 & 13 & 39 & 4.33 \\
\hline 52 & Aldehyde dehydrogenase & ELA36993.1 & 49.96 & 6.25 & 6 & 16 & $\infty$ \\
\hline 68 & Malate dehydrogenase & ELA31304.1 & 34.33 & 6.98 & 9 & 32 & 6.61 \\
\hline 58 & Aminoglycoside phosphotransferase & ELA31403.1 & 41.71 & 6.19 & 21 & 59 & 2.88 \\
\hline 69 & Dienelactone hydrolase family protein & ELA32769.1 & 30.97 & 6.12 & 5 & 15 & 2.47 \\
\hline 60 & Short chain dehydrogenase & ELA24821.1 & 35.86 & 8.11 & 9 & 26 & 4.02 \\
\hline 63 & Superoxide dismutase & ELA31430.1 & 23.00 & 7.04 & 4 & 21 & $\infty$ \\
\hline 65 & 3-hydroxyanthranilate 3,4-dioxygenase & ELA32139.1 & 17.72 & 5.58 & 3 & 22 & $\infty$ \\
\hline 45 & CipC-like antibiotic response protein & ELA27578.1 & 13.82 & 6.09 & 10 & 63 & 3.80 \\
\hline 46 & CipC-like antibiotic response protein & ELA27578.1 & 13.82 & 6.09 & 3 & 24 & $\infty$ \\
\hline 47 & CipC-like antibiotic response protein & ELA27578.1 & 13.82 & 6.09 & 5 & 43 & $\infty$ \\
\hline 59 & Aminoglycoside phosphotransferase & ELA31403.1 & 41.71 & 6.19 & 16 & 52 & 4.21 \\
\hline \multicolumn{8}{|c|}{ UNKNOW } \\
\hline 48 & Uncharacterized protein & ELA37769.1 & 12.73 & 5.91 & 4 & 22 & $\infty$ \\
\hline 55 & Minor allergen alt a 7 & ELA26079.1 & 26.48 & 6.03 & 4 & 13 & 2.01 \\
\hline
\end{tabular}

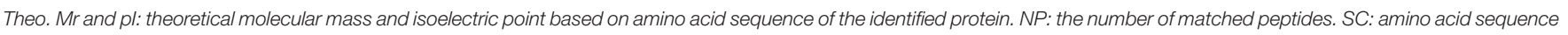

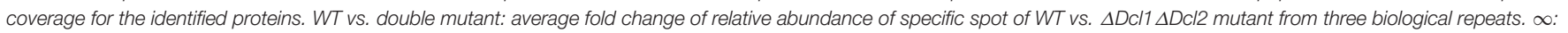
corresponding spot appeared in the WT but not in the $\Delta D c / 1 \Delta D c / 2$ mutant.

\section{DCLs Affect Gene Transcript Levels}

To determine whether DCLs regulated the proteins abundance by affecting the transcript levels, the quantitative RT-PCR analysis was conducted. Our results showed that the relative levels of all of the 16 selected genes were significantly decreased; and the results was consistent with that of 2D analysis (Figure 7). These data suggested that the DCLs could directly function by affecting the transcript levels.

\section{DISCUSSION}

Alignment of amino acid sequences showed that the sequence of DCL of C. gloeosporioides has a high identity with that of $C$. higginsianum, $N$. crassa, and B. cinerea, indicating that the DCLs are well conserved in filamentous fungi (Figure S1). In the present study, knocking out of Dcl1 or Dcl2 did not affect the growth rate of C. gloeosporioides in vitro; but the double deletion mutant
$\Delta \mathrm{Dcl} 1 \Delta \mathrm{Dcl} 2$ showed an obvious reduction of growth rate. Conidiation is important for the reproduction and pathogenicity of fungi. Deletion of Dcl2 did not influence the conidiation process; while deletion of $D c l 1$ greatly improved the conidiation; and the loss of the two genes caused a tremendous reduction of the conidiation. These results suggested that the DCLs were important for the normal vegetative growth and conidiation of C. gloeosporioides. Furthermore, only the double mutant showed severe impair on the normal growth and conidiation, suggesting functional redundancy between DCL1 and DCL2.

Anthracnose of rubber tree mainly occurred at the tender and wounded leaves (Cai et al., 2013). In the present study, the pathogenicity of the C. gloeosporioides was accessed by inoculating the conidia of the WT and mutants to the detached "light green" leaves without wound. The results showed that $\Delta \mathrm{Dcl} 1 \Delta \mathrm{Dcl} 2$ caused no lesion on the leaves of Hevea brasiliensis, indicating the complete loss of pathogenicity; whereas both $\Delta \mathrm{Dcl} 1$ and $\Delta \mathrm{Dcl} 2$ showed similar pathogenicity as WT. The 


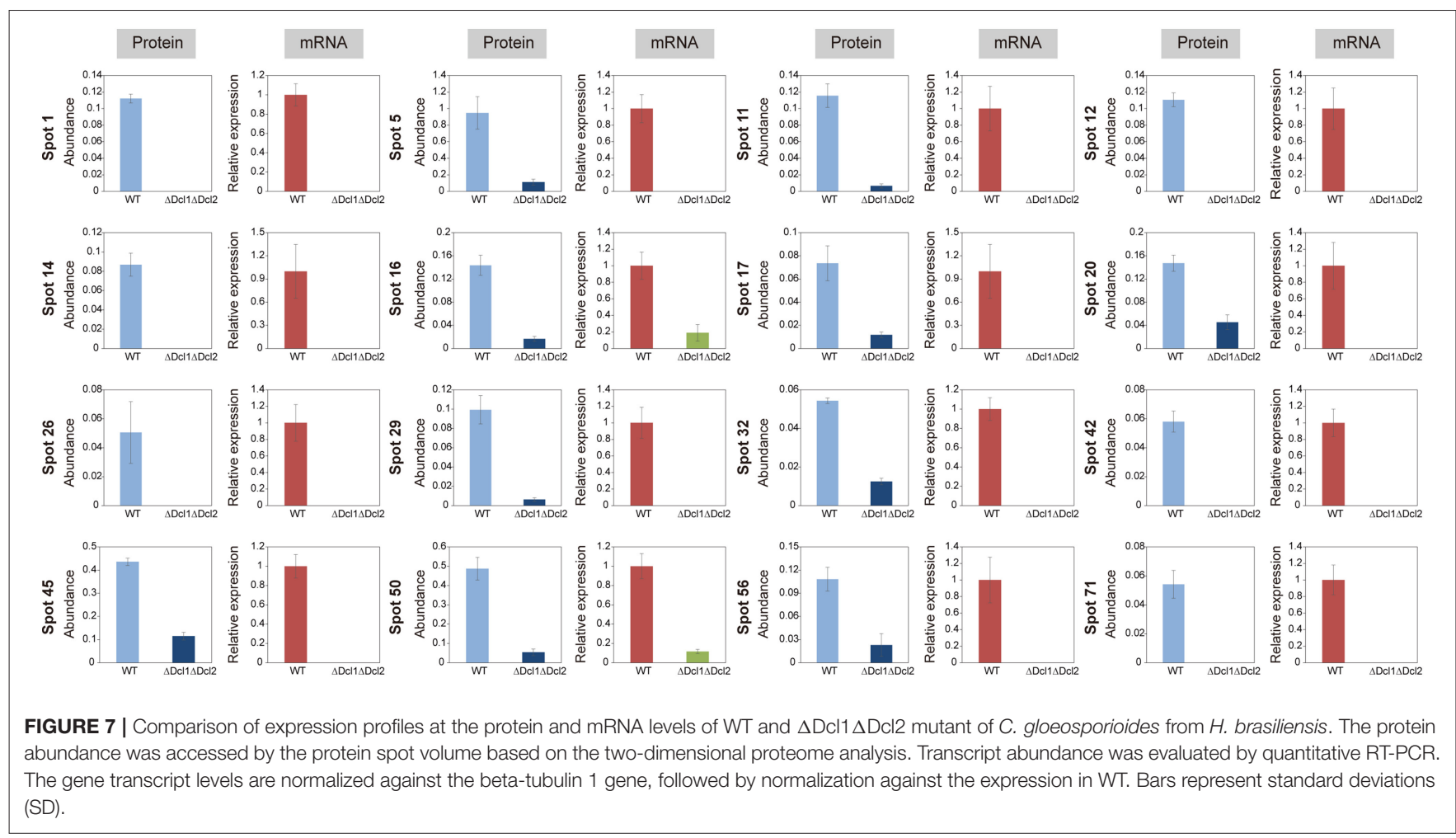

conidia viability and successful penetration are critical steps for many fungal pathogens to infect host plants. Our results showed that the germination rate of conidia of $\Delta \mathrm{Dcl} 1 \Delta \mathrm{Dcl} 2$ mutant was nearly same as the WT when cultured on the surface of cellophane with sufficient nutrient (Figures 4B,C). When inoculated on onion epidermis, conidia of the WT could successfully penetrate into the onion epidermis after $12 \mathrm{~h}$ and formed complex networks in the onion cells after $20 \mathrm{~h}$; but those of $\Delta \mathrm{Dcl} 1 \Delta \mathrm{Dcl} 2$ mutant did not geminate at all after $12 \mathrm{~h}$ and only grow on the surface instead of penetrating into the onion cells after $20 \mathrm{~h}$ (Figure 4A). These results suggest that loss of penetration ability was the main cause for the decreased pathogenicity of the $\Delta \mathrm{Dcl} 1 \Delta \mathrm{Dcl} 2$ mutant.

Oxidative burst is one of earliest events in the plant hypersensitive response to the pathogen attack. In the present study, DAB staining was used to analyze the hydrogen peroxide accumulation. The results showed that the mechanical damage could induce a slightly peroxide accumulation in CK. When inoculated with the WT strain of C. gloeosporioides, there was a significantly increase of hydrogen peroxide all over the leaves; but the leaves inoculated with $\Delta \mathrm{Dcl} 1 \Delta \mathrm{Dcl} 2$ did not induce the oxidative burst, indicating that the interaction between the host and the pathogen was also impaired in $\Delta \mathrm{Dcl} 1 \Delta \mathrm{Dcl} 2$.

Small RNAs were proved to play multiple functions in living cells, including the regulation of interaction between plant and pathogens (Padmanabhan et al., 2009; Li et al., 2013; Pumplin and Voinnet, 2013; Ouyang et al., 2014). In order to identify the potential targets of DCLs in C. gloeosporioides cells, proteome profiles of the wild type and $\Delta \mathrm{Dcl} 1 \Delta \mathrm{Dcl} 2$ mutant were analyzed. Using the 2D gel electrophoresis, a total of 72 proteins spots were identified to be down-regulated in the $\Delta$ Dcl1 $\Delta$ Dcl2 mutant. First, 6 spots representing 2 proteins related to cell cycle and 12 spots represented 9 proteins related to proteins synthesis were down regulated in the double mutant. Protein biosynthesis are the basis for normal cell growth and cell division. The decrease in expression of these two categories of proteins caused the significant impair on the hyphae growth and cell division, which lead to the depression of vegetative growth and conidiation processed (Figure 2). Chaperones are required for macromolecules to fold correctly and perform their normal biological functions. In the study, we identified 5 proteins related to polypeptide stability and folding, indicating the complicated influences of DCLs on the protein synthesis. Second, abundance of 6 proteins involved in the transport and 3 cell structure proteins were significantly reduced. Among them, the Rab GTPase, Ras GTPases and clathrin were all reported to be involved in the vesicle transport (Salminen and Novick, 1987; Dumas et al., 2001; Gall et al., 2002); protein disulfide-isomerase and alpha-mannosidase are involved in the modification of secreted proteins; actin also participate in the substance transport, in addition to its function in cytoskeleton formation (Gottlieb et al., 1993). According to the previous report, impair of the vesicle transport could also induced the decrease of the cell growth, development, and the pathogenicity of pathogens (Zhang et al., 2014), which were in accordance with our results (Figures 2, 3). It is well known that, in order to facilitate the penetration process, fungal pathogens could secret abundance of hydrolytic enzymes to degrade the cell wall or defense 
proteins of the host plant. Here we found that 4 hydrolytic enzymes, including aspergillopepsin-2, endochitinase, aspartic endopeptidase and proteasome endopeptidase, were decreased in the double mutant, which may impair the penetration ability of the pathogen (Figure 4). 5 proteins (8 spots) related to signal transduction were also down regulated in the double mutant, indicating that DCLs also function as signal regulators. Moreover, 9 identified proteins are related to metabolisms were also downregulated, including tricarboxylic acid cycle, alcohol metabolism, fatty-acid and isoprenoid metabolism, superoxide metabolism and antibiotic response proteins, indicating the diverse functions of the DCLs. Taking together, the proteomics assays revealed that DCLs regulate vegetative growth and conidiation by delaying the cell cycle, repress the protein synthesis and even the disturbance of the cell skeleton. Fungal pathogenicity is up to many biological processes, including the attachment of conidia to host plant surface, conidia germination, hyphal penetration, and overcoming of plant immunity. In the present study, a series of proteins directly related to or involved in the fungal pathogenicity were significantly down regulated, which may be the main cause of the loss of pathogenicity of the mutant. It has been reported that DCLs could affect the transcript levels of the target genes via generation of small RNAs (Li et al., 2013; Ouyang et al., 2014). In the present study, the quantitative RT-PCR assays showed that the transcript levels of 16 selected genes were significantly decreased, and results were in consistent with the variation in abundance of relative protein, suggesting that the DCLs regulated

\section{REFERENCES}

An, B., Li, B., Li, H., Zhang, Z., Qin, G., and Tian, S. (2016). Aquaporin8 regulates cellular development and reactive oxygen species production, a critical component of virulence in Botrytis cinerea. New Phytol. 209, 1668-1680. doi: $10.1111 / \mathrm{nph} .13721$

Bartel, D. P. (2004). MicroRNAs: genomics, biogenesis, mechanism, and function. Cell 116, 281-297. doi: 10.1016/S0092-8674(04)00045-5

Bass, B. L. (2000). Double-stranded RNA as a template for gene silencing. Cell 101, 235-238. doi: 10.1016/S0092-8674(02)71133-1

Blevins, T., Rajeswaran, R., Shivaprasad, P. V., Beknazariants, D., Si-Ammour, A., Park, H. S., et al. (2006). Four plant dicers mediate viral small RNA biogenesis and DNA virus induced silencing. Nucleic Acids Res. 34, 6233-6246. doi: 10.1093/nar/gkl886

Cai, Z., Li, G., Lin, C., Shi, T., Zhai, L., Chen, Y., et al. (2013). Identifying pathogenicity genes in the rubber tree anthracnose fungus Colletotrichum gloeosporioides through random insertional mutagenesis. Microbiol. Res. 168, 340-350. doi: 10.1016/j.micres.2013.01.005

Carthew, R. W., and Sontheimer, E. J. (2009). Origins and mechanisms of miRNAs and siRNAs. Cell 136, 642-655. doi: 10.1016/j.cell.2009.01.035

Daudi, A., Cheng, Z., O’Brien, J. A., Mammarella, N., Khan, S., Ausubel, F. M., et al. (2012). The apoplastic oxidative burst peroxidase in Arabidopsis is a major component of pattern-triggered immunity. Plant Cell 24, 275-287. doi: 10.1105/tpc.111.093039

Ding, S. W., and Lu, R. (2011). Virus-derived siRNAs and piRNAs in immunity and pathogenesis. Curr. Opin. Virol. 1, 533-544. doi: 10.1016/j.coviro.2011.10.028

Dumas, B., Borel, C., Herbert, C., Maury, J., Jacquet, C., Balsse, R., et al. (2001). Molecular characterization of CLPT1, a SEC4-like Rab/GTPase of the phytopathogenic fungus Colletotrichum lindemuthianum which is regulated by the carbon source. Gene 272, 219-225. doi: 10.1016/S0378-1119(01)00 $536-4$ protein abundance via affecting the transcript levels of relative genes.

To summarize, DCLs regulate the growth, conidiation and pathogenicity by affecting the expression of a series of functional proteins in C. gloeosporioides.

\section{AUTHOR CONTRIBUTIONS}

QW and BA conceived and designed this study. QW, XH, and YG performed the experiments. BA and HL wrote the manuscript. $\mathrm{CH}$ provided critical advice. All authors approved the final manuscript to be published.

\section{FUNDING}

This study was supported by the National Natural Science Foundation of China (No. 31560044), the National Natural Science Foundation of China (No. 31360424), the Natural Science Foundation of Hainan Province (No. 20163046), the startup funding and the scientific research foundation of Hainan University (No. kyqd1517).

\section{SUPPLEMENTARY MATERIAL}

The Supplementary Material for this article can be found online at: https://www.frontiersin.org/articles/10.3389/fmicb. 2017.02621/full\#supplementary-material

Gall, W. E., Geething, N. C., Hua, Z., Ingram, M. F., Liu, K., Chen, S. I., et al. (2002). Drs2p-dependent formation of exocytic clathrin-coated vesicles in vivo. Curr. Bio. 12, 1623-1627. doi: 10.1016/S0960-9822(02)01148-X

Göhre, V., and Robatzek, S. (2008). Breaking the barriers: microbial effector molecules subvert plant immunity. Annu. Rev. Phytopathol. 46, 189-215. doi: 10.1146/annurev.phyto.46.120407.110050

Gottlieb, T. A., Ivanov, I. E., Adesnik, M., and Sabatini, D. D. (1993). Actin microfilaments play a critical role in endocytosis at the apical but not the basolateral surface of polarized epithelial cells. J. Cell Biol. 120, 695-710. doi: $10.1083 /$ jcb.120.3.695

Jones, J. D. G., and Dangl, J. L. (2006). The plant immune system. Nature 444, 323-329. doi: 10.1038/nature05286

Kleemann, J., Rincon-Rivera, L. J., Takahara, H., Neumann, U., van Themaat, E. V. L., van der Does, H. C., et al. (2012). Sequential delivery of hostinduced virulence effectors by appressoria and intracellular hyphae of the phytopathogen Colletotrichum higginsianum. PLoS Pathog. 8:e1002643. doi: 10.1371/journal.ppat.1002643

Koeck, M., Hardham, A. R., and Dodds, P. N. (2011). The role of effectors of biotrophic and hemibiotrophic fungi in infection. Cell. Microbiol. 13, 1849-1857. doi: 10.1111/j.1462-5822.2011.01665.x

Lee, Y. S., Nakahara, K., Pham, J. W., Kim, K., He, Z., Sontheimer, E. J., et al. (2004). Distinct roles for Drosophila Dicer-1 and Dicer-2 in the siRNA/miRNA silencing pathways. Cell 117, 69-81. doi: 10.1016/S0092-8674(04)00261-2

Li, S., Liu, L., Zhuang, X., Yu, Y., Liu, X., Cui, X., et al. (2013). MicroRNAs inhibit the translation of target mRNAs on the endoplasmic reticulum in Arabidopsis. Cell 153, 562-574. doi: 10.1016/j.cell.2013.04.005

MacRae, I. J., Zhou, K., Li, F., Repic, A., Brooks, A. N., Cande, W. Z., et al. (2006). Structural basis for double-stranded RNA processing by Dicer. Science 311, 195-198. doi: 10.1126/science.1121638

Matzke, M. A., and Matzke, A. J. (1995). How and why do plants inactivate homologous (trans) genes? Plant Physiol. 107, 679-685. 
Mendell, J. T., and Olson, E. N. (2012). MicroRNAs in stress signaling and human disease. Cell 148, 1172-1187. doi: 10.1016/j.cell.2012. 02.005

Moazed, D. (2009). Small RNAs in transcriptional gene silencing and genome defence. Nature 457, 413-420. doi: 10.1038/nature07756

Nolan, T., Braccini, L., Azzalin, G., De Toni, A., Macino, G., and Cogoni, C. (2005). The post-transcriptional gene silencing machinery functions independently of DNA methylation to repress a LINE1-like retrotransposon in Neurospora crassa. Nucleic Acids Res. 33, 1564-1573. doi: 10.1093/nar/gki300

O'Connell, R. J., Thon, M. R., Hacquard, S., Amyotte, S. G., Kleemann, J., Torres, M. F., et al. (2012). Lifestyle transitions in plant pathogenic Colletotrichum fungi deciphered by genome and transcriptome analyses. Nat. Genet. 44, 1060-1065. doi: 10.1038/ng.2372

Ouyang, S., Park, G., Atamian, H. S., Han, C. S., Stajich, J. E., Kaloshian, I., et al. (2014). MicroRNAs suppress NB domain genes in tomato that confer resistance to Fusarium oxysporum. PLoS Pathog. 10:e1004464. doi: 10.1371/journal.ppat.1004464

Padmanabhan, C., Zhang, X., and Jin, H. (2009). Host small RNAs are big contributors to plant innate immunity. Curr. Opin. Plant Biol. 12, 465-472. doi: 10.1016/j.pbi.2009.06.005

Pumplin, N., and Voinnet, O. (2013). RNA silencing suppression by plant pathogens: defence, counter-defence and counter-counter-defence. Nat. Rev. Microbiol. 11, 745-760. doi: 10.1038/nrmicro3120

Rafiqi, M., Ellis, J. G., Ludowici, V. A., Hardham, A. R., and Dodds, P. N. (2012). Challenges and progress towards understanding the role of effectors in plant-fungal interactions. Curr. Opin. Plant Biol. 15, 477-482. doi: 10.1016/j.pbi.2012.05.003

Romano, N., and Macino, G. (1992). Quelling: transient inactivation of gene expression in Neurospora crassa by transformation with homologous sequences. Mol. Microbiol. 6, 3343-3353. doi: 10.1111/j.1365-2958.1992.tb02202.x

Rubio-Somoza, I., Cuperus, J. T., Weigel, D., and Carrington, J. C. (2009). Regulation and functional specialization of small RNA-target nodes during plant development. Curr. Opin. Plant Biol. 12, 622-627. doi: 10.1016/j.pbi.2009.07.003

Salminen, A., and Novick, P. J. (1987). A ras-like protein is required for a post-Golgi event in yeast secretion. Cell 49, 527-538. doi: 10.1016/0092-8674(87)90455-7

Schmitz, R. J., Hong, L., Fitzpatrick, K. E., and Amasino, R. M. (2007). DICERLIKE 1 and DICER-LIKE 3 redundantly act to promote flowering via repression of FLOWERING LOCUS C in Arabidopsis thaliana. Genetics 176, 1359-1362. doi: 10.1534/genetics.107.070649

Stephenson, S. A., Hatfield, J., Rusu, A. G., Maclean, D. J., and Manners, J. M. (2000). CgDN3: an essential pathogenicity gene of Colletotrichum gloeosporioides necessary to avert a hypersensitive-like response in the host Stylosanthes guianensis. Mol. Plant Microbe Interact. 13, 929-941. doi: 10.1094/MPMI.2000.13.9.929

Suárez, Y., Fernández-Hernando, C., Pober, J. S., and Sessa, W. C. (2007). Dicer dependent microRNAs regulate gene expression and functions in human endothelial cells. Circ. Res. 100, 1164-1173. doi: 10.1161/01.RES.0000265065.26744.17

Volpe, T. A., Kidner, C., Hall, I. M., Teng, G., Grewal, S. I., and Martienssen, R. A. (2002). Regulation of heterochromatic silencing and histone H3 lysine-9 methylation by RNAi. Science 297, 1833-1837. doi: 10.1126/science.1074973

Weiberg, A., Wang, M., Lin, F. M., Zhao, H., Zhang, Z., Kaloshian, I., et al. (2013). Fungal small RNAs suppress plant immunity by hijacking host RNA interference pathways. Science 342, 118-123. doi: 10.1126/science.1239705

Xue, Z., Yuan, H., Guo, J., and Liu, Y. (2012). Reconstitution of an Argonautedependent small RNA biogenesis pathway reveals a handover mechanism involving the RNA exosome and the exonuclease QIP. Mol. Cell 46, 299-310. doi: 10.1016/j.molcel.2012.03.019

Yang, Q., Jiang, J., Mayr, C., Hahn, M., and Ma, Z. (2013). Involvement of two type $2 \mathrm{C}$ protein phosphatases $\mathrm{BcPtc} 1$ and $\mathrm{BcPtc} 3$ in the regulation of multiple stress tolerance and virulence of Botrytis cinerea. Environ. Microbiol. 15, 2696-2711. doi: 10.1111/1462-2920.12126

Zhang, Z., Qin, G., Li, B., and Tian, S. (2014). Knocking out Bcsas1 in Botrytis cinerea impacts growth, development, and secretion of extracellular proteins, which decreases virulence. Mol. Plant Microbe Interact. 27, 590-600. doi: 10.1094/MPMI-10-13-0314-R

Conflict of Interest Statement: The authors declare that the research was conducted in the absence of any commercial or financial relationships that could be construed as a potential conflict of interest.

Copyright (C) 2018 Wang, An, Hou, Guo, Luo and He. This is an open-access article distributed under the terms of the Creative Commons Attribution License (CC BY). The use, distribution or reproduction in other forums is permitted, provided the original author(s) or licensor are credited and that the original publication in this journal is cited, in accordance with accepted academic practice. No use, distribution or reproduction is permitted which does not comply with these terms. 